\title{
Comparative Analysis of European and American Standards for Maximum Fault Current Calculations on Medium Voltage Mine Power Networks
}

\author{
Adam Heyduk ${ }^{1}$, Jaroslaw Joostberens ${ }^{1}$ \\ ${ }^{I}$ Silesian University of Technology, Faculty of Mining and Geology, Department of Electrical \\ Engineering and Control in Mining, \\ Akademicka 2A St. 44-100, Gliwice, Poland \\ adam.heyduk@polsl.pl
}

\begin{abstract}
Continuous work of mine supplying electrical power networks is a prerequisite to ensure the continuity of the mining process and the safety of its crew. The choice of the appropriate method for calculating short-circuit currents is therefore particularly important in terms of both economic and safety considerations. The methods used are always a compromise between the accuracy of obtained results, computational complexity and availability of data necessary for calculations. The study compares the two most common standards for calculating maximum short-circuit currents - IEC and ANSI/IEEE. The study presents the most important interrelationships that describe the characteristic magnitudes of short-circuit currents for IEC standard and for the ANSI/IEEE standard. This comparison has been done on the basis of novel calculations performed on a typical MV mine network. The results of the calculations showed a high contiguity of the results despite the different methodological assumptions for rotating machines modelling and for determining the equivalent value of resistance and reactance of the network where the fault occurred, and the extent of assumed simplifications.
\end{abstract}

Index Terms-Short-circuit currents; standards; power distribution; mining industry; equivalent circuits.

\section{INTRODUCTION}

The increase in the number and power of drives installed in the mining industry, with the concurrent increase of quality requirements concerning power supply leads to changes in the structure of power systems supplying industrial plants and individual receivers. These changes are usually associated with a reduction of impedance of the power supply network and the increase in value of shortcircuit currents. This leads to more stringent requirements regarding switchgear and other elements of the power supply network. Hence the need to improve the methods for calculating the maximum short-circuit currents. The complexity of the electromagnetic and thermal phenomena accompanying short-circuits in electrical power systems, associated with quantitative and qualitative descriptions of transients in electrical machines and electrical apparatus

Manuscript received 8 August, 2015; accepted 4 February, 2016. makes it more difficult to directly use these descriptions in engineering practice when making technical decisions, with their important economic impact. This is related to investment costs of switching, measuring, protection devices and other elements of the power system. Which leads to the necessity of creating simplified, easy to apply procedures, ensuring sufficient accuracy for specific needs. These methods are contained in the standard recommendations which have been created in many countries over several decades. The first such standard was the introduced in Germany in 1929 VDE0102 standard. Currently the most widely used - also in Poland - is the international IEC 60909 standard [1]. Given the current globalization of manufacturing processes and the use of electrical industry products, it seems interesting (in both scientific and practical terms) to make a comparison of the provisions of this standard with the standards applied in the United States. Such comparison - which is the subject of this study - can lead to better comprehension of the fundamental differences between the two approaches (being developed in parallel over a dozen years) and detailed assessment of the impact of these differences on the results obtained using different calculation procedures. The question of short-circuit current calculation is described in several standards [2]-[5], the fundamental one being the oldest of them [2], while the other documents are its repetitions with additions and extensions, especially [4], [5] - which describe the issues of calculating short-circuits in systems with static converters. European standard covers the calculation of both the maximum and minimum values of short-circuit currents. The calculation procedures contained in the American standard cover essentially only the maximum short-circuit currents necessary for the selection of switching devices, since they constitute only a part of a broader standard describing the parameters and requirements for medium-voltage switchgear - as it was the case with the Polish standard [6]. Issues of calculating the minimum short-circuit currents and selecting of safety settings are presented in separate standards [4], [5]. Comparisons of these standards have already been the 
subject of several papers [7], [8], however, the starting point was always the ANSI standard, and calculation examples related rather to American conditions. This study presents the basic assumptions of both standards and an example of calculation similar to the existing in Polish conditions MV mine network. It should be also emphasized that both the European [1] and American [3]-[5] have the status of voluntary use (in the U.S. standard defined as IEEE Recommended Practice). This is an important difference compared to the earlier Polish standard [6] whose application was mandatory. Currently, it is mandatory to use only [9], which means that the rules for calculating the minimum short-circuit currents contained in IEC standard are not being applied with respect to mine networks. From a formal point of view, the choice of method of calculation of the maximum short-circuit current is therefore not imposed by any legal act. Therefore, the choice of the method of calculating the maximum short-circuit currents is left to the designer. Due to Poland's membership in the EU it became natural to adopt methodology for calculation of the maximum short-circuit currents specified in [1]. It is interesting and appropriate to compare the results of shortcircuit calculations carried out by the IEC method with the results obtained by ANSI method, in particular with respect to the mine network, characterized by a defined specificity. Some standard descriptions and comparisons can be found in [10] and in textbooks [11], [12] but they refer to general power distribution networks, not particularly to industrial ones. In [13]-[15] there are some numerical examples but they refer mainly to synchronous generators used in power plants and are based on dynamical simulation (EMTP-like programs) and not on standard-recommended simplified analytical formulas.

\section{THE MAIN DIFFERENCES BETWEEN THE EUROPEAN AND AMERICAN STANDARD}

The IEC standard defines the voltage of a equivalent source with the use of voltage factor $c$ of the assumed value depending on the network nominal voltage and on the purpose of calculation in accordance with Table I.

TABLE I. VALUES OF VOLTAGE FACTORS RECOMMENDED IN [1]
\begin{tabular}{|c|c|c|}
\hline \multirow{2}{*}{ Nominal voltage $\boldsymbol{U}_{\boldsymbol{n}}$} & \multicolumn{2}{|c|}{$\begin{array}{c}\text { Voltage factor } \boldsymbol{c} \\
\text { for calculation of }\end{array}$} \\
\cline { 2 - 3 } & $\begin{array}{c}\text { maximum short- } \\
\text { circuit current }\end{array}$ & $\begin{array}{c}\text { minimum short- } \\
\text { circuit current }\end{array}$ \\
\hline $\begin{array}{c}\text { Low voltage: } \\
100 \mathrm{~V} \text { to } 1000 \mathrm{~V}\end{array}$ & 1,05 & 0,95 \\
\hline $\begin{array}{c}\text { Medium voltage } \\
\text { (up to } 35 \mathrm{kV})\end{array}$ & 1,10 & 1,0 \\
\hline High voltage $(>35 \mathrm{kV})$ & 1,1 & 1,0 \\
\hline
\end{tabular}

For calculation of minimum short-circuit currents in mine networks applies the standard [8].

In calculations according to [1] the fundamental role plays the concept of initial symmetrical short-circuit current $I_{k}^{\prime \prime}$ (defined as the as the rms value of the periodic component of the short-circuit current at time $t=0$ and determined from the following equation:

$$
I_{k}^{\prime \prime}=\frac{c \times U_{n}}{\sqrt{3} \times \sqrt{R_{k}^{2}+X_{k}^{2}}},
$$

where $c$-voltage factor (Table I), $U_{n}$ - nominal voltage of the network where the assumed for calculation short-circuit has occurred $\mathrm{kV}, R_{k}$ - short-circuit resistance $\Omega, X_{k}$ - shortcircuit reactance $\Omega$.

In [2]-[5] the equivalent of the initial symmetrical shortcircuit current $I_{k}{ }^{\prime \prime}$ is the so called first cycle duty, defined as the maximum calculative value of the symmetrical shortcircuit current in the first cycle after the occurrence of shortcircuit and is calculated as

$$
I_{\text {sym }}=\frac{E}{X},
$$

where $E$ - rms (nominal) value of line-to-neutral voltage $\mathrm{kV}$, $X$ - circuit reactance value at the time of short-circuit $\Omega$.

When calculating the current values of the first cycle in medium voltage networks [2] takes into account only the reactances of individual network elements, while considering both reactance and resistance is recommended for lowvoltage networks [4], [5]. Peak short-circuit current $i_{p}$ [1], that is the largest instantaneous value of short-circuit current is calculated from the equation

$$
i_{p}=\kappa \times \sqrt{2} \times I_{k}^{\prime \prime}
$$

where $\kappa$-factor for the calculation of the peak short-circuit current.

Factor $\kappa$ can be also calculated from the equation or read from the graph $\kappa=f(R / X)$ or else $\kappa=f(X / R)$, contained in [1].

The equivalent of the peak short-circuit current $i_{p}$ in [2] is the peak current $I_{\text {peak }}$ defined as

$$
I_{\text {peak }}=\sqrt{2} \times I_{\text {sym }} \times\left(1+e^{-\frac{2 \pi \tau}{(X / R)}}\right),
$$

where $\tau$ - relative time from the moment of short-circuit to the maximum current value (in relation to the network voltage cycle).

Relative time $\tau$ is calculated from

$$
\tau=0,49-0,1 e^{-\frac{(X / R)}{3}},
$$

Estimation of the peak current value is done in two stages. The first step is to determine the value of the relative time $\tau$ (it will be always be a little less than half of the cycle), and the next one is to calculate the value of $I_{\text {peak }}$ current. The question arises as to what extent the formulas that describe the currents in a network of $60 \mathrm{~Hz}$ frequency can be used in networks with a frequency of $50 \mathrm{~Hz}$. However, it can be 
noted here that the factor $e^{-(R / L) t}$ occurring in the equation (independent of frequency) describing the process of disappearance of the aperiodic component, contains inductance $L$ as $L=X / \omega=X \times T /(2 \pi)$, and time $t$ as a multiple of the network's cycle, that is $t=N \times T$ where $T$ is the length of the network's cycle. This leads to $e^{-(2 \pi R /(X \times T)) N \times T}=e^{-2 \pi N /(X / R)}$ that is to the result which is independent of frequency. Of course, the ratio $X / R$ must be set at the frequency for which the calculation is carried out, that is, in the actual case, of $50 \mathrm{~Hz}$.

To determine the ratio $X / R$ [2] recommends the separate setting of equivalent network's reactance (while omitting all the equivalent resistances of individual elements of the network), and assigning equivalent network's resistance while omitting all reactances. This avoids the calculations using complex numbers and it is justified by the fact that in any case the time-consuming calculation of the resultant short-circuit impedance does not lead to the correct value of the $\mathrm{X} / \mathrm{R}$ ratio, since in a network supplied from several sources, each source branch is characterized by a distinct value of the time constant of decay of aperiodic component, while the simplified method ensures that the resulting peak current values are not lower than the real ones, or provides sufficient accuracy for the proper selection of switchgear.

The symmetrical short-circuit breaking current is the rms value of the periodic component of the short-circuit current at the time of disconnection of the connector contacts. Calculation of symmetrical short-circuit breaking current (according to [1]) requires a distinction of the type of shortcircuit. In case of a short-circuit near-to-generator (or motor) the symmetrical breaking short-circuit current has the value lower than the initial short-circuit current. The symmetrical short-circuit breaking current in case of a near-to-generator short-circuit is calculated according to

$$
I_{b G}=\mu_{G} \times I_{k G}^{\prime \prime}
$$

where $I_{k G}$ " - Initial breaking current from the generator kA, $\mu_{G}-$ Factor for the calculation of the symmetrical shortcircuit breaking current.

Occurring in (6) factor $\mu_{G}$ depends on the ratio of currents: an initial short-circuit coming from the generator referred to the rated current of the generator $I_{k G}^{\prime \prime} / I_{r G}$ and the minimum time delay $\left(t_{\min }\right)$. The values of $\mu_{G}$ factor can be read from the graph contained in [1]. In this standard, for selected minimum time delays, are also given formulas to calculate the $\mu_{G}$ factor. When the minimum time delay differs from the graph values, it is acceptable to use linear interpolation between the curves of the graph so as to determine the correct values of $\mu_{G(M)}$ factor. The symmetrical short-circuit breaking current from the induction motor is calculated from

$$
I_{b M}=\mu_{M} \times q \times I_{k M}^{\prime \prime}
$$

The $\mu_{M}$ factor is determined likewise, as in the case of synchronous generators, with the difference that the equation includes also the factor $q$ for the calculation of breaking current of asynchronous motors and the ratio $I_{k G}^{\prime \prime} / I_{r G}$ is replaced by $I_{k M}^{\prime \prime} / I_{r M}$. The value of the factor q depends on engine power $\left(P_{r M}\right)$ per pair of poles $(p)$ expressed in MW and on minimum time delay. It is possible to determine the $q$ factor by reading its value from a graph or by calculation using the equations contained in [1].

Short-circuit in a network supplied via a transformer can be considered as a far-from-generator short-circuit if the following condition is met

$$
X_{T K} \geq 2 X_{Q}^{\prime}
$$

where $X_{T K}$ - corrected reactance of the transformer $\Omega, X_{Q}{ }^{\prime}-$ equivalent reactance of the transformer supply network $\Omega$.

Both reactances from the condition (8) should be converted to voltage of the network in which the computational fault location was assumed - in the case of calculations on MV mine networks, usually on the lower voltage side of the transformer. When the condition (8) is met, the symmetric short-circuit breaking current is equal to the initial short-circuit current. According to [2]-[5], in case of a synchronous generator the short-circuit can be regarded as far-from-generator when any of the two following conditions are met:

1. reactance between the generator and the place of the short-circuit exceeds by one and a half the value of subtransient reactance of the generator,

2. there is more than one transformer between the generator and the place of short-circuit.

In [2], the equivalent to symmetrical short-circuit breaking current $I_{b}$ is the so called interrupting current $I_{s c}$ determined in the similar way as the first cycle duty, but with the recalculated (in relation to the values assumed in the calculation of the $I_{\text {sym }}$ current value) reactance values of rotating machines. The use of appropriate reactance multipliers maps the phenomenon of gradual disappearance of electromotive forces in synchronous and induction machines (Figs. $1 \& 2$ ). The values of multipliers, relevant to MV mine network elements, are shown in Table II (the power values are presented in the SI system, and the velocity values related to the frequency of $50 \mathrm{~Hz}$ ).

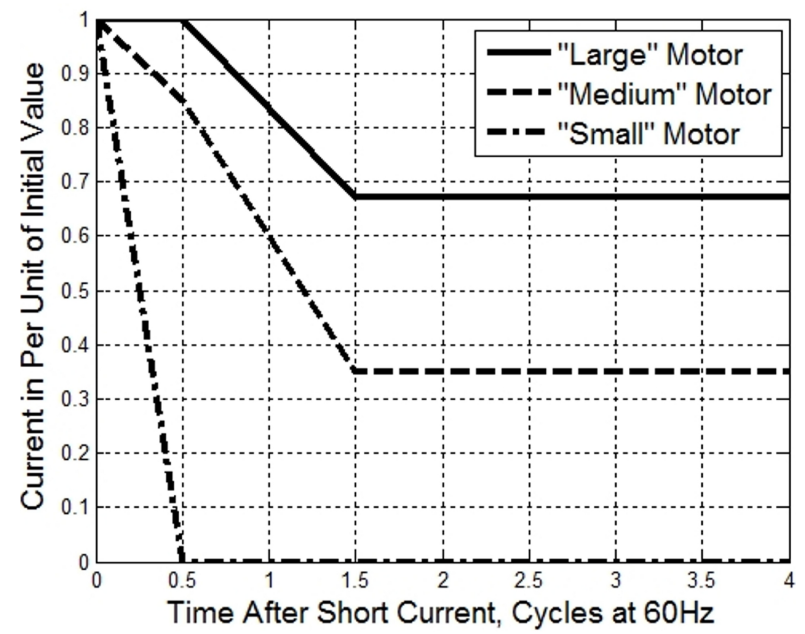

Fig. 1. Simplified course of decay of a current generated by induction motors according to [2], [4]. 


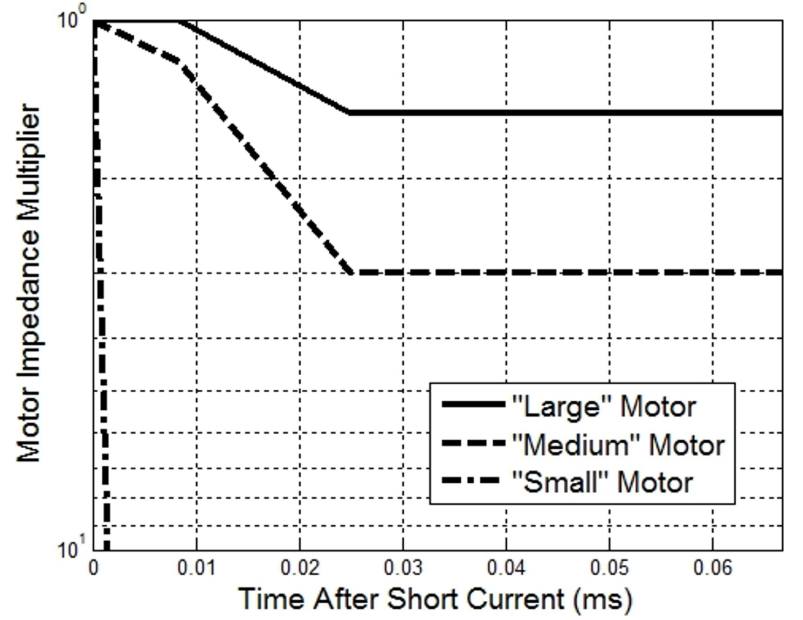

Fig. 2. Multipliers of Motors' Impedance corresponding to decay from Fig. 1.

TABLE II. VALUES OF REACTANCE MULTIPLIERS TO CALCULATE THE CURRENT OF THE FIRST CYCLE AND BREAKING CURRENT

\begin{tabular}{|c|c|c|}
\hline Criterion & $\begin{array}{c}\text { Multipliers for } \\
\text { calculating the first } \\
\text { cycle duty }\end{array}$ & $\begin{array}{l}\text { Multipliers for } \\
\text { calculating the } \\
\text { breaking current }\end{array}$ \\
\hline \multicolumn{3}{|c|}{ Synchronous machines } \\
\hline turbogenerators & 1,0 & 1,0 \\
\hline synchronous motors & 1,0 & 1,5 \\
\hline \multicolumn{3}{|c|}{ Induction machines } \\
\hline $\begin{array}{c}>735,5 \mathrm{~kW} \text { and } \leq 1500 \\
\text { turns } / \mathrm{min}\end{array}$ & 1,0 & 1,5 \\
\hline $\begin{array}{c}>183.9 \mathrm{~kW} \text { and } 3000 \\
\text { turns } / \mathrm{min}\end{array}$ & 1,0 & 1,5 \\
\hline$\geq 36.8 \mathrm{~kW}$ & 1,2 & 3 \\
\hline$<36.8 \mathrm{~kW}$ & $\infty$ & $\infty$ \\
\hline
\end{tabular}

Asymmetric breaking current is the geometric sum of initial short-circuit current and short-circuit current's aperiodic component. The value of d.c. (aperiodic) component of short-circuit current at time $t$ is calculated from the following equation [1]

$$
i_{\text {d.c. }}=\sqrt{2} \times I_{k}^{\prime \prime} \times e^{-2 \pi f t \frac{R_{k}}{X_{k}}},
$$

where $f$ - nominal frequency $\mathrm{Hz}$.

According to [2] asymmetric breaking current $I_{\text {asym }}$ is determined on the basis of symmetric current and a multiplier $\mathrm{M}$ which value depends on two parameters:

- minimum time delay (presented as the number of cycles $C$ ),

$-X / R$ ratio in the place where short-circuit occurred.

This multiplier values are selected on the basis of the graphs contained in [2]. It should be noted that separate graphs describe the value of $M_{R}$ multiplier the in case of farfrom- generator short-circuit (where disappears only the aperiodic component) and $M_{L}$ for near-to-generator shortcircuit (aperiodic component disappears, while the periodic component decreases). For remote short-circuits this multiplier can be evaluated from the formula

$$
M_{R}=\sqrt{1+2 e^{-\frac{4 \pi C}{(X / R)}}} .
$$

In case when short-circuit at a specific point in the network is supplied from both distant and nearby sources, it is proposed to interpolate the multiplier $\mathrm{M}$ using the following formula [2], [4]

$$
M=\left(M_{R}-M_{L}\right) \times N A C D+M_{L},
$$

where $M$ - resultant (interpolated) value of multiplier, $M_{R}-$ multiplier value for remote short-circuits, $M_{L}$ - multiplier value for local short-circuits, $N A C D$ - ratio of the sum of currents from remote sources (No AC Decay) to summary short-circuit current $I_{\text {sym }}$.

In that case the value of asymmetric breaking current is calculated as follows

$$
I_{\text {asym }}=M \times I_{\text {sym }} .
$$

\section{CALCUlation OF MAXIMUM SHORT-CiRCUIT CURRENTS IN OPEN NETWORK SUPPLIED FROM SEVERAL INDEPENDENT SOURCES}

In compliance with the recommendations contained in [1], the short-circuit calculations should be done by setting the equivalent impedance $\underline{Z}_{k}$ using the equivalent network diagram transformation. Impedances of the individual elements, which are connected via a transformer to the network where the short-circuit location was assumed for calculation, must be converted to a voltage of the network. The initial short-circuit current in short-circuit location $F$ is the geometric sum of component short-circuit currents

$$
\underline{I}_{k}^{\prime \prime}=\sum_{i} \underline{I}_{k i}^{\prime \prime}
$$

In the case of a short circuit in an open network supplied from several sources it is allowed to calculate the initial short-circuit current at the fault location $F$, as the algebraic sum of the individual component short-circuit currents, which can by expressed by the following formula

$$
I_{k}^{\prime \prime} \approx \sum_{i} I_{k i}^{\prime \prime}
$$

When calculating the maximum short-circuit currents, all the generators connected to the network affected by a shortcircuit must be assumed as the source of short-circuit current, and there should be evaluated the participation of synchronous and induction motors powered from the network (either directly or through a transformer) in which the fault location was assumed for calculation.

In compliance with [1], in calculations of the maximum short-circuit currents, the synchronous motors should be considered as synchronous generators, therefore to these machines apply the same principles of calculation and formulas that apply to generators. In relation to the MV network, the medium voltage induction motors must be taken into account in the calculation of the maximum shortcircuit current [1], thus they should be regarded as sources of short-circuit current. 
In the case of an open network supplied from several independent sources, the short-circuit peak current at the fault location $F$ is determined as the sum of the individual short-circuit peak current components

$$
i_{p}=\sum_{i} i_{p i}
$$

The symmetric short-circuit breaking current is calculated in the similar way, treating it as the sum of the component currents flowing from various sources

$$
I_{b}=\sum_{i} I_{b i}
$$

\section{EQuivalent IMPEDANCES OF SELECTED NeTWORK ELEMENTS}

Equivalent impedance (as a complex number) of the supply network can be can be written as

$$
\underline{Z}_{Q}=R_{Q}+j X_{Q}
$$

It is determined on the basis of the initial short-circuit current at the supply location $I_{k}{ }^{\prime \prime} \mathrm{kA}$ or the initial symmetrical short-circuit power $S_{k}$ " MVA. The equivalent impedance of the supply network (expressed as a complex number) can be calculated from one of the following equations:

$$
\underline{Z}_{Q}=\frac{c U_{n Q} \times\left(R_{Q} / X_{Q}+j 1\right)}{\sqrt{3} \times I_{k Q}^{\prime \prime} \sqrt{1+\left(R_{Q} / X_{Q}\right)^{2}}},
$$

or

$$
\underline{Z}_{Q}=\frac{c U_{n Q}^{2} \times\left(R_{Q} / X_{Q}+j 1\right)}{S_{k}^{\prime \prime} \sqrt{1+\left(R_{Q} / X_{Q}\right)^{2}}}
$$

where $c$ - voltage factor; $c=1.1, U_{n Q}-$ nominal network voltage at the point of connection $Q \mathrm{kV}, R_{Q} / X_{Q}$ - resistance to reactance ratio in equivalent network.

In the case of high-voltage power supply network $\left(U_{n Q}>\right.$ $35 \mathrm{kV}$ ), with the unknown $R_{Q} / X_{Q}$ ratio, it is assumed that $R_{Q} \approx 0 \Omega$.

Equivalent impedance of a two-winding transformer is calculated based on the nominal parameters of the transformer. In accordance with the recommendations of [1], calculation of short-circuit currents requires multiplying the value of equivalent impedance of the transformer by the correction factor $K_{T}$, which can be written as follows

$$
\underline{Z}_{T K}=K_{T} \times\left(R_{T}+j X_{T}\right),
$$

where $R_{T}$ - equivalent resistance of the transformer $\Omega, X_{T}-$ equivalent reactance of the transformer $\Omega, K_{T}$ - impedance correction factor for two-winding network transformer.

The required $K_{T}$ factor correcting transformer's parameters is calculated from the following

$$
K_{T}=0.95 \frac{c_{\max }}{1+x_{T} \times 0.6}
$$

where $c_{\max }$ - voltage factor (Table I), $x_{T}$ - relative reactance of the transformer, $x_{T}=X_{T} /\left(U_{r T}^{2} / S_{r T}\right)$.

Thus the corrected equivalent impedance (expressed as a complex number) can be determined from

$$
\underline{Z}_{T K}=K_{T} \times \frac{u_{R r}}{100} \times \frac{U_{r T}^{2}}{S_{r T}} \times\left(1+j \sqrt{\left(\frac{u_{k r}}{u_{R r}}\right)^{2}-1}\right),
$$

where $u_{R r}$ - rated resistive component of the short-circuit voltage of a transformer in per cent, $u_{k r}$ - rated short-circuit voltage of transformer in per cent, $U_{r T}$ - rated voltage of transformer on the high-voltage or low-voltage side $\mathrm{kV}, S_{r T}-$ rated apparent power of the transformer MVA.

When calculating short-circuit currents according to [1], the impedance of a synchronous generator, similarly to transformer's impedance, should be multiplied by the correction factor $K_{G}$. Then the equivalent impedance of a synchronous generator can be written in the following form

$$
\underline{Z}_{G K}=K_{G} \times\left(R_{G}+j X_{d}^{\prime \prime}\right)
$$

where $R_{G}-$ equivalent resistance of synchronous generator $\Omega, X_{d}$ " - subtransient reactance of the synchronous generator $\Omega$.

Correction factor for equivalent impedance of synchronous generator (synchronous motor) $K_{G(S)}$ is calculated from

$$
K_{G(S)}=\frac{U_{n}}{U_{r G(S)}} \times \frac{c_{\max }}{1+x_{d}^{\prime \prime} \times \sin \phi_{r G(S)}},
$$

where $U_{r G(S)}$ - rated voltage of the generator (synchronous motor) $\mathrm{kV}, x_{d}{ }^{\prime}$-relative subtransient reactance of the generator (motor), $x_{d}{ }^{\prime \prime}=X_{d}{ }^{\prime \prime} /\left(U_{r G(S)}^{2} / S_{r G(S)}\right), \varphi_{r G(S)}$ - phase angle between $\underline{I}_{r G}$ and $U_{r G(S)} / \sqrt{3}, S_{r G(S)}$ - rated apparent power of the generator (motor) MVA.

To determine the impedance $\underline{Z}_{G(S) K}$ is necessary to know the rated data of the machine and compared $R_{G(S)} / X_{d}$ " for the synchronous generator (synchronous motor). Appropriate $R_{G(S)} / X_{d}$ " ratios and the criteria for their selection are presented in Table III.

Corrected equivalent impedance of synchronous generator (synchronous motor) can be derived from the following

$$
\underline{Z}_{G(S) K}=K_{G(S)} \times \frac{X_{d \%}^{\prime \prime}}{100} \times \frac{U_{r G(S)}^{2}}{S_{r G(S)}} \times\left(\frac{R_{G(S)}}{X_{d}^{\prime \prime}}+j 1\right),
$$

where $R_{G(S)} / X_{d}{ }^{\prime}$ - value selected from Table III.

In the case of synchronous motors, with the knowledge of the rated power $P_{r S}$ (on the shaft of the machine), (25) takes the following form 


$$
\underline{Z}_{S K}=K_{S} \times \frac{X_{d \%}^{\prime \prime}}{100} \times \frac{U_{r S}^{2} \times \cos \phi_{r S} \times \eta_{r S}}{P_{r S}} \times\left(\frac{R_{S}}{X_{d}^{\prime \prime}}+j 1\right),
$$

where $P_{r S}$ - rated active power of synchronous motor MW, $U_{r S}-$ rated voltage of synchronous motor $\mathrm{kV}, \cos \varphi_{r S}-$ rated power factor, $\eta_{r S}$ - rated efficiency of the synchronous motor, $R_{S} / X_{d}$ " - value selected from Table III.

The equivalent impedance (expressed as complex number) of the induction motor used for calculation of maximum short-circuit currents can be presented by the following formula

$$
\underline{Z}_{M}=R_{M}+j X_{M}
$$

Its evaluation is made on the basis of rated values of the induction machine and the $R_{M} / X_{M}$ ratio. The formula for the equivalent impedance of the induction motor can be expressed as follows

$$
\underline{Z}_{M}=\frac{1}{I_{L R} / I_{r M}} \times \frac{U_{r M}^{2} \times \cos \phi_{r M} \times \eta_{r M}}{P_{r M} \sqrt{1+\left(R_{M} / X_{M}\right)^{2}}} \times\left(\frac{R_{M}}{X_{M}}+j 1\right)
$$

where $P_{r M}$ - rated active power of asynchronous motor, MW, $U_{r M}$ - rated voltage of asynchronous motor $\mathrm{kV}, I_{L R} / I_{r M}$ - ratio of the locked-rotor to the rated current of the motor, $\cos \varphi_{r M}$ - rated power factor, $\eta_{r M}$ - rated efficiency of the asynchronous motor, $R_{M} / X_{M}$ - value selected from Table III.

\begin{tabular}{|c|c|c|}
\hline \multicolumn{3}{|c|}{ WITH [1]. } \\
\hline Criterion & $R_{G(S) / X} X_{d}$ & $R_{M} / X_{M}$ \\
\hline \multicolumn{3}{|c|}{ Synchronous generators } \\
\hline$U_{r G}>1 \mathrm{kV}$ and $S_{r G} \geq 100 \mathrm{MVA}$ & 0.05 & $\mathrm{X}^{2}$ \\
\hline$U_{r G}>1 \mathrm{kV}$ and $S_{r G}<100 \mathrm{MVA}$ & 0.07 & \\
\hline$U_{r G} \leq 1 \mathrm{kV}$ & 0.15 & 3 \\
\hline \multicolumn{3}{|c|}{ Induction machines } \\
\hline$U_{r M}>1 \mathrm{kV}$ and $P_{r M} / p \geq 1 \mathrm{MW}$ & $\rightarrow$ & 0.10 \\
\hline$U_{r M}>1 \mathrm{kV}$ and $P_{r M} / p<1 \mathrm{MW}$ & 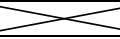 & 0.15 \\
\hline$U_{r M} \leq 1 \mathrm{kV}$ & 3 & 0.42 \\
\hline
\end{tabular}

TABLE III. VALUES OF $R_{M} / X_{M}$ AND $R_{G} / X_{D}$ ” RATIOS FOR CALCULATION OF SHORT-CIRCUIT CURRENTS IN COMPLIANCE

Equivalent impedance of the short-circuit current-limiting reactor $Z_{R}$ is basically equal to its reactance $X_{R}$, because $R_{R}$ $<<X_{R}$. Its value can be calculated from the following

$$
X_{R}=\frac{u_{k R}}{100} \times \frac{U_{r R}}{\sqrt{3} \times I_{r R}},
$$

where $u_{k R}$ - short-circuit voltage of a short-circuit limiting reactor in per cent, $U_{r R}$ - rated voltage of the reactor $\mathrm{kV}, I_{r R}$ - rated current of the reactor $\mathrm{kA}$.

Equivalent impedance of the cable line used for calculation of the maximum short-circuit currents can be derived from the following

$$
\underline{Z}_{L}=R_{L}+j X_{L}=l \times\left(R_{L}^{\prime}+j X_{L}^{\prime}\right),
$$

where $R_{L}{ }^{\prime} ; X_{L}{ }^{\prime}$ - unit resistance and reactance of the cable line $\Omega / \mathrm{km}, l$ - length of cable line $\mathrm{km}$.

The ANSI/IEEE standards do not provide for the use of the factors correcting impedance of transformers and synchronous machines (as defined in [1]). But there should be applied appropriate (separate for first cycle current and breaking current) reactance multipliers for synchronous motors and induction machines (Table II). Supply network impedance is determined for the value $c=1$.

\section{CAlCulation ExAmPle}

To illustrate the differences in the calculation of the maximum short-circuit current according to IEC and on the other hand ANSI/IEEE standards, there were conducted calculations of characteristic short-circuit currents, necessary for the selection of $W$ switch on the sample segment of mine power system. Diagram of electric power network under consideration (with the rating of the individual elements, used to calculate the impedance) is shown in Fig. 3, and selected according to both standards parameters of individual elements in Table IV.

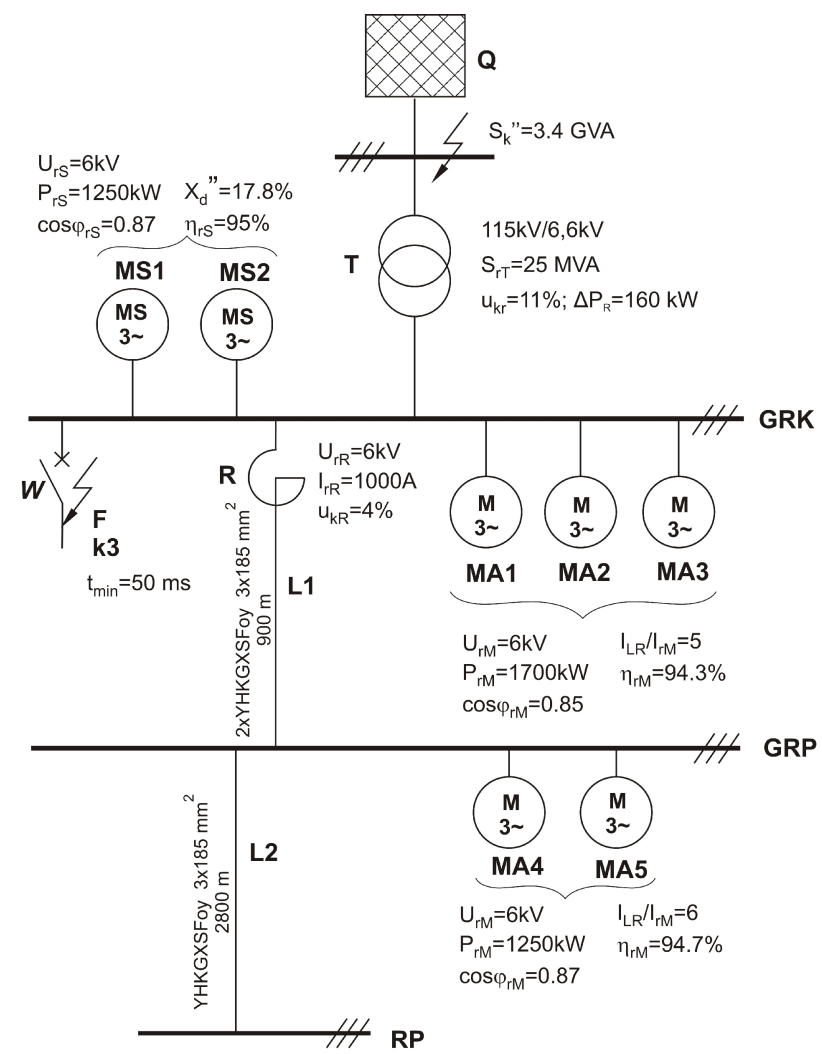

Fig. 3. Diagram of an exemplary network for comparing calculations according to IEC and ANSI/IEEE standards (GRK - main mine switchboard, GRP - main level switchboard, RP - intermediate switchboard, Q - power supply network, T - transformer, MA1 - MA5 asynchronous motors, MS1, MS2 - synchronous motors, L1, L2 - cable lines, $\mathrm{R}$ - short-circuit limiting reactor, $\mathrm{W}$ - circuit-breaker).

Equivalent diagram for the calculation of the maximum short-circuit currents (according to [1]) with the assumed for computation fault location (as in Fig. 3) is shown in Fig. 4. This diagram may also be used to calculate the maximum short-circuit current according to [2]-[5], assuming the values of the factors $K_{T}=K_{S}=1$ and $c=1$. The values of equivalent impedances of different elements of the network are shown in Table IV.

The initial short-circuit current was determined through the conversion of the network diagram. Following the principles contained in [1] concerning calculation of short- 
circuit currents in an open network supplied from several independent sources, there have been also calculated the component initial short-circuit currents.

TABLE IV. IMPEDANCE VALUES OF INDIVIDUAL ELEMENTS OF THE SYSTEM SHOWN IN FIGURE 3 CHOSEN ACCORDING TO [1]

\begin{tabular}{|c|c|c|c|c|c|c|}
\multicolumn{2}{c|}{} & \multicolumn{2}{|c|}{ IEC } & \multicolumn{4}{c|}{ ANSI/IEEE } \\
\cline { 2 - 8 } $\begin{array}{c}\text { Network } \\
\text { element }\end{array}$ & $\mathbf{R}$ & $\mathbf{X}$ & \multicolumn{2}{c|}{$\begin{array}{c}\text { for calculating } \\
\text {,first cycle current" }\end{array}$} & $\begin{array}{c}\text { for calculating } \\
\text { „breaking } \\
\text { current" }\end{array}$ \\
\cline { 4 - 8 } & $\Omega$ & $\Omega$ & $\mathbf{R}(\boldsymbol{\Omega})$ & $\mathbf{X}(\boldsymbol{\Omega})$ & $\mathbf{R}(\boldsymbol{\Omega})$ & $\mathbf{X}(\boldsymbol{\Omega})$ \\
\hline $\mathrm{Q}$ & - & 0.013 & - & 0.012 & - & 0.012 \\
\hline T & 0.011 & 0.188 & 0.011 & 0.191 & 0.011 & 0.191 \\
\hline MS1, MS2 & 0.217 & 3.107 & 0.213 & 3.044 & 0.213 & 4.566 \\
\hline $\begin{array}{c}\text { MA1, MA2, } \\
\text { MA3 }\end{array}$ & 0.502 & 3.357 & 0.502 & 3.357 & 0.502 & 5.035 \\
\hline MA4,MA5 & 0.585 & 3.911 & 0.585 & 3.911 & 0.585 & 5.867 \\
\hline R & - & 0.139 & - & 0.139 & - & 0.139 \\
\hline L1 & 0.089 & 0.077 & 0.089 & 0.077 & 0.089 & 0.077 \\
\hline
\end{tabular}

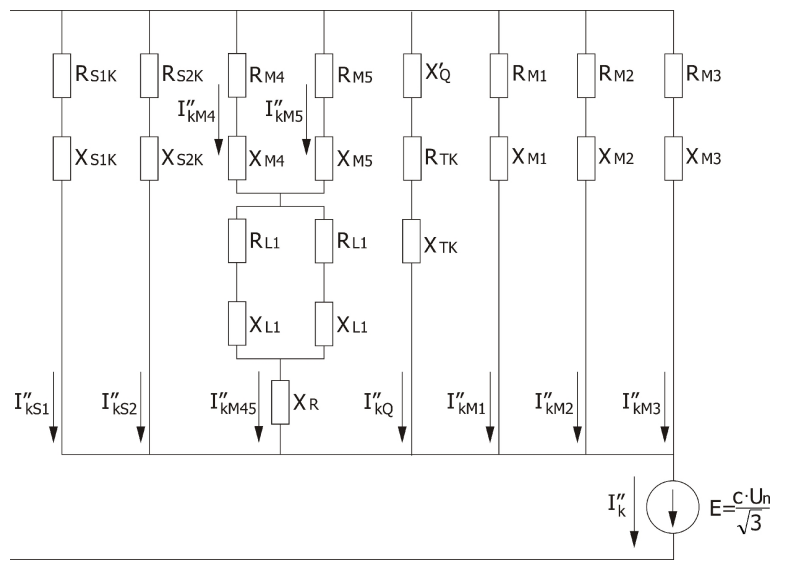

Fig. 4. Equivalent diagram of a short-circuited circuit (for positivesequence component).

The diagrams for calculating equivalent network reactance and resistance according to [2] is shown in Fig. 5 and Fig. 6.

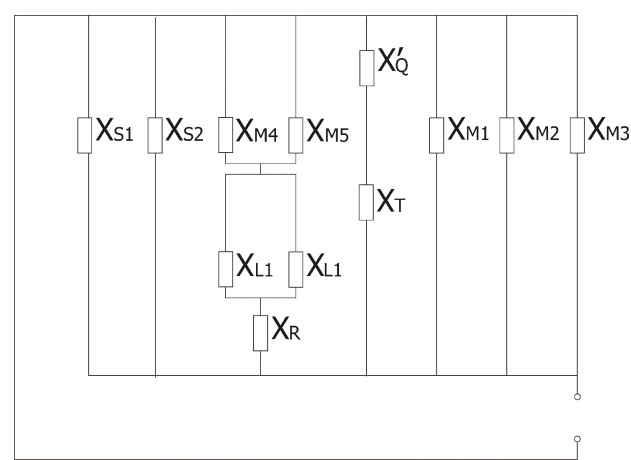

Fig. 5. The diagram of reactance network used to determine the shortcircuit current and the value of $\mathrm{X} / \mathrm{R}$ ratio in the calculations compatible with [2].

In conformity with these principles, the other characteristic short-circuit currents were calculated, and the results were tabulated (Table V). This table also contains the results of short-circuit currents calculations conducted in accordance with ANSI/IEEE standard. It should be noted that the value of the aperiodic component $I_{s c}$ was determined for comparative purposes, as a geometric difference $\sqrt{I_{\text {asym }}^{2}-I_{s c}^{2}}$, since [2] does not provide for its direct determination. The values of $I_{\text {sym }}, I_{\text {peak }}$ components were determined only for comparative purposes, since [2] does not provide for their direct calculation (with an exception of the cases when it is necessary to determine the value of $N A C D$ ratio and interpolation of multiplier $M$ ). The value of $\mathrm{X} / \mathrm{R}$ ratio as determined by a method of separate transformation of the reactive and resistive networks amounts to 15.5. By way of comparison it can be indicated that after the transformation the impedance diagram (as in [1]) the obtained result amounted to $X / R=13.4$. On the other hand the value of $N A C D$ factor used in the interpolation of multipliers for calculating the current $I_{\text {asym }}$ in the considered example was determined as

$$
N A C D=I_{\text {sym } Q} / I_{\text {sym }}=17.1 / 24.1=0.71 .
$$

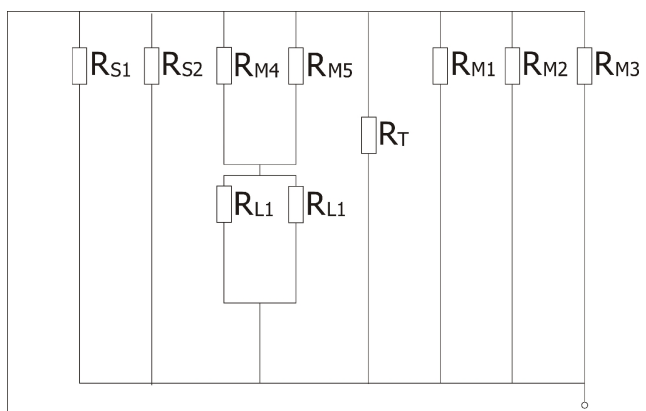

Fig. 6. The diagram of resistance network used to determine and the value of $\mathrm{X} / \mathrm{R}$ ratio in the calculations compatible with [2].

TABLE V. THE RESULTS OF CALCULATIONS OF MAXIMUM SHORT-CIRCUIT CURRENTS IN THE EXAMPLE SHOWN ON FIG. 3.

\begin{tabular}{|c|c|c|c|c|c|c|c|c|c|c|}
\hline \multirow{2}{*}{ 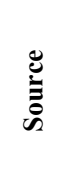 } & \multicolumn{5}{|c|}{$\begin{array}{l}\text { according to ANSI/IEEE } \\
\text { standards }\end{array}$} & \multicolumn{5}{|c|}{ according to IEC standard } \\
\hline & $I_{\text {sym }}$ & $I_{\text {peak }}$ & $I_{s c}$ & $I_{d c}$ & $I_{\text {asym }}$ & $I_{k i}$ & $i_{p i}$ & $\boldsymbol{I}_{b i}$ & $i_{\text {d.c. }}$ & $I_{\text {basym }}$ \\
\hline & \multicolumn{5}{|c|}{$\mathrm{kA}$} & \multicolumn{5}{|c|}{$\mathrm{kA}$} \\
\hline Q & 17.1 & 44.5 & 17.1 & - & - & 19.0 & 49.7 & 19.0 & - & - \\
\hline MS1 & 1.1 & 2.9 & 0.8 & - & - & 1.2 & 3.1 & 1.0 & - & - \\
\hline MS2 & 1.1 & 2.9 & 0.8 & - & - & 1.2 & 3.1 & 1.0 & - & - \\
\hline MA1 & 1.0 & 2.4 & 0.7 & - & - & 1.1 & 2.6 & 0.7 & - & - \\
\hline MA2 & 1.0 & 2.4 & 0.7 & - & - & 1.1 & 2.6 & 0.7 & - & - \\
\hline MA3 & 1.0 & 2.4 & 0.7 & - & - & 1.1 & 2.6 & 0.7 & - & - \\
\hline MA4 & 0.8 & \multirow{2}{*}{3.8} & 0.6 & - & - & 0.9 & \multirow{2}{*}{4.1} & 0.5 & - & - \\
\hline MA5 & 0.8 & & 0.6 & - & - & 0.9 & & 0.5 & - & - \\
\hline$\Sigma$ & 23.9 & 61.3 & 22.0 & 10.4 & 24.3 & 26.5 & 67.8 & 24.1 & 11.6 & 26.7 \\
\hline $1.1 \cdot \Sigma$ & 26.3 & 67.4 & 24.2 & 11.4 & 26.9 & & & & & \\
\hline
\end{tabular}

\section{CONCLUSIONS}

Due to the economic aspects of switchgear selection and related to ensuring safety in underground mines requirements concerning continuity of supply, the problem of calculating the maximum short-circuit currents is of particular importance. It is reasonable, therefore, to compare short-circuit calculations carried out by different methods. Comparative analysis of the calculation results point to the fact that the calculation of all the currents according to [2] lead to results lower by about $10 \%$ from the results of calculations carried out according to [1]. This is mostly 
related to the use of the factor stipulated in [1], which is not used according to [2]. After taking into account this fact (ie, scaling the obtained results) discrepancies between these standards do not exceed $1.5 \%$. But interesting differences can be noticed when individual motor contributions to symmetrical breaking current are analysed. In the case of induction motors (MA1...MA5) current values according to ANSI standard are higher than according to IEC standard. This is contrary to the case of synchronous motors (MS1\&MS2) where contributions according to IEC are higher than contributions according to ANSI. It should be also noted that [2]-[5] are much simpler computationally (basically do not require calculations performed on complex numbers, as they only consider network reactances, and do not require correction of impedance of transformers and synchronous generators). The depicted example shows that quite different (simplified) modelling methodology of disappearance of the components from rotating machines through the use of appropriate impedance multipliers - leads to very similar results.

\section{REFERENCES}

[1] Int Std IEC 60909-0, Short-Circuit Currents in Three-Phase AC Systems Part 0: Calculation of currents. 2002.

[2] ANSI/IEEE Std C37.010, IEEE Application Guide for AC High Voltage Circuit Breakers Rated on a Symmetrical Current Basis. 1979.

[3] ANSI/IEEE Std 141, IEEE Recommended Practice for Electric Power Distribution for Industrial Plants (Red Book), 1993.

[4] ANSI/IEEE Std 399, IEEE Recommended Practice for Industrial and Commercial Power Systems Analysis (Brown Book), 1997.
[5] ANSI/IEEE Std 551, IEEE Recommended Practice for Calculating Short-Circuit Currents in Industrial and Commercial Power Systems (Violet Book), 2006.

[6] PN-74/E-05002 Electrical Power Equipment. Selection of Highvoltage Apparatuses Depending on Short-circuit Conditions, 1974

[7] G. Knight, H. Sieling "Comparison of ANSI and IEC 909 Shortcircuit current calculation procedures", IEEE Trans. on Industry Applications, vol. 29, pp. 625-630, 1993. [Online]. Available: http://dx.doi.org/10.1109/28.222435

[8] A. J. Rodolakis, "A Comparison of North American (ANSI) and European (IEC) fault calculation guidelines", IEEE Trans. Industry Applications, vol. 29, pp. 515-521, 1993. [Online]. Available: http://dx.doi.org/10.1109/28.222420

[9] PN-G-42042, Protective and Safety Measures in the Mining Power Networks - Short-circuit and Overload Protection - Requirements and Rules for Selection, 1998.

[10] D. Nedic, G. Bathurst, J. Heath, "A comparison of short circuit calculation methods and guidelines for distribution networks", in Proc. 19 th Int. Conf. on Electricity Distribution, Vienna, 2007.

[11] N. D. Tleiss, Power Systems Modelling and Fault Analysis. Theory and Practice. Elsevier, 2008.

[12] J. C. Das, Power system analysis short-circuit load flow and harmonics. BocaRaton FL: CRC Press, 2011. [Online]. Available: http://dx.doi.org/10.1201/b11021

[13] C. G. Kaloudas, P. N. Papadopoulos, T. A. Papadopoulos, "Shortcircuit analysis of an isolated generator and comparative study of IEC, ANSI and dynamic simulation", in Proc. $7^{\text {th }}$ Mediterranean Conf. and Exhibition on Power Generation, Transmission, Distribution and Energy Conversion MedPower, Agia Napa 2010. [Online]. Available: http://dx.doi.org/10.1049/cp.2010.0922

[14] T. A. Papadopoulos, C. G. Kaloudas, A. G. Marinopoulos, "Static and dynamic calculation of short-circuit currents in synchronous generators", in Proc. Int. Conf. Power Systems Transients, Delft 2011.

[15] S. L. Shankar, M. M. Iqbal, "ANSI and IEC standards based short circuit analysis of a typical $2 \times 30 \mathrm{mw}$ thermal power plant", MiddleEast J. Sci. Res., vol. 23, no. 8, 2015. 